ISSN: $1130-3743$

\title{
DOCENTES NOVELES UNIVERSITARIOS Y SU ENSEÑANZA CON TIC
}

\section{Beginning university teachers and his teaching with ICT}

\section{Nouvelles enseignants universitaires et son enseignement}

avec TIC

Joaquín PAREDES LABRA

Facultad de Formación de Profesorado y Educación. Departamento de Didáctica y Teoría de la Educación. Campus de Cantoblanco. C/ Francisco Tomás y

Valiente, 3. 28049 Madrid.Correo-e: joaquin.paredes@uam.es

Fecha de recepción: enero de 2012

Fecha de aceptación definitiva: abril de 2012

Biblid [(1130-3743) 24, 1-2012, 133-150]

\section{RESUMEN}

Se analiza la percepción de la integración de las Tic en la enseñanza universitaria por parte de docentes universitarios noveles. Se ha utilizado un protocolo y observación participante con recogida de evidencias en forma de productos de enseñanza. Entre los principales resultados se encuentra que la enseñanza que practican es tradicional, que perciben la enseñanza como una suma de técnicas, que los procesos de cambio que vive la universidad europea no les presionan, que no han reflexionado suficientemente sobre la práctica de enseñanza y que las Tic son un elemento de sus prácticas que tiene un carácter secundario en las mismas. Se observa cómo el peso de concepciones tradicionales de la enseñanza condiciona muy negativamente las posibilidades de integración de las Tic y la metodología que desarrollará cada docente. Se ofrecen algunas recomendaciones para el cambio.

Palabras clave: universidad, TIC, uso de las Tic en educación, formación de docentes en activo, desarrollo profesional docente, profesor novel. 


\section{SUMMARY}

We analyze the perception of the integration of ICT in higher education from beginning scholars. It uses a protocol and participant observation with collection of evidences in the form of educational products. Among the major findings we meet that teaching practice is traditional, teaching is perceived as a sum of techniques, processes of change that are experienced in European universities do not push them, have not reflect enough about teaching, and that ICT is one element of their practice that has not the main role. It shows how traditional education negatively affects the possibilities of integration of ICT and the methodology each teacher uses. It offers some recommendations for change.

Key words: higher education, ICT, educational uses of ICT, inservice teacher education, teacher professional development beginning teacher.

\section{SOMMAIRE}

Nous analysons la perception de l'intégration des TIC dans l'enseignement universitaire des nouveaux enseignants. Nous avons utilisé un protocole et d'observation participante à la collecte de preuves sous forme de produits éducatifs. Parmi les principales conclusions est que l'enseignement qu'on a observé est traditionnel, qui considèrent l'éducation comme une somme de techniques, que le procés de changement de l'enseignement universitaire à l'Europe ne les puisse pas, n'ont pas suffisamment réfléchi sur les pratiques pédagogiques et de que les Tic sont un élément de leurs pratiques qui ont un caractère secondaire par eux. Nous avons observé que le poids des conceptions traditionnelles de l'enseignement influe négativement sur les possibilités d'intégration des Tic et de la méthodologie développée par chaque enseignant. Il inclut des recommandations pour le changement.

Mots clés: université, TIC, utilisation des TIC dans l'éducation, formation des enseignants en exercice, développement professionnel des enseignants, nouvel enseignant.

\section{INTRODUCCIÓN}

¿En qué consiste ser profesor universitario? Hanna (2002) dice que es una mezcla de libertad académica (capacidad de expresión), autogestión (control profesional), presencia local de políticas institucionales (agente de la universidad en un campus que es espacio de aprendizaje), trabajo instrumental y trabajo de experto.

Moacir Gadotti (2005), compañero de Paulo Freire, dice de los docentes que hacen fluir el saber, dan sentido a la vida de las personas y buscan, con el resto de las personas, un mundo más justo, más productivo y más saludable para todos, lo que a buen seguro también son atributos de los docentes universitarios. 
Observamos dos concepciones de la enseñanza, la última totalizadora y orientada a fines y la de Hanna (2002), funcional y compartimentalizada lo que, como se dirá, es algo connatural a la profesión de docente universitario.

El estudio de la enseñanza en la universidad viene siendo una constante desde finales de los años ochenta (con alguna propuesta pionera, como los trabajos del profesor Fernández Pérez, 1989), interés que se ha avivado con el proceso de convergencia de enseñanzas universitarias en Europa (VV. AA., 2000; García Valcárcel, 2001; Zabalza, 2003), y se ha concretado en la actualidad en una constante y rica producción de informes de investigación financiados por la administración educativa española.

Se ha denunciado la forma de procurar desarrollo profesional a los docentes en la universidad. Enseñar en la universidad parece una técnica o una suma de técnicas (Alba, 2005; Gewerc, 2008), y a esto se mantienen fieles las tenues políticas que observamos en diversas universidades, como se verá más adelante como estrategias utilizada para la formación inicial.

A propósito de la convergencia metodológica de las universidades europeas (el Espacio Europeo de Educación Superior, EEES) cobra interés en la enseñanza universitaria la introducción de las TIC, con abundante literatura sobre los procesos institucionales (por ejemplo, VV. AA., 2009), lo que se convierte en otro desafío para todos los docentes, incluidos los noveles.

La universidad vive, pues, su particular encrucijada, una intersección de temáticas sobre una enseñanza universitaria renovada donde los docentes en ejercicio oyen hablar de reformas, innovación y cambios, y donde los noveles se han de incorporar.

En este trabajo, que se interesa por la forma de llegar a ser docente universitario de algunos de ellos, denominamos profesores noveles en la universidad a docentes recién incorporados a la plantilla de una universidad, generalmente con contratos de formación (ayudantes, ayudantes doctores), aunque pueden ser contratados doctores (quienes generalmente deben haber pasado por los contratos previos) o profesores asociados (quienes en puridad son profesionales sin formación como docentes). También hay personal investigador en formación, entre cuyas obligaciones está su colaboración en la docencia, y cuyas becas y contratos son el escalón natural previo a un contrato de formación de los primeros. Todos ellos son responsables de alguna materia.

El estudio de los profesores noveles hunde sus raíces en los años sesenta y se recupera en los ochenta, y contamos con excelentes revisiones, como la de Marcelo (2008), si bien se nos habla del docente de los niveles no universitarios y generalmente del docente de Primaria. En estos estudios hay, por tanto, un espacio para la reflexión sobre la práctica docente que en otros niveles ya se ha recorrido, pero que en la docencia universitaria conviene pues parece que la enseñanza no es la primera de las tareas de los docentes universitarios.

¿Qué ocurre con la enseñanza de los profesores noveles universitarios? ¿Qué necesitan los profesores noveles para afrontar su trabajo? ¿Cuánto de los procesos 
de cambio les llega, les afecta, les presiona? ¿Los docentes noveles pueden formar parte de este proceso de cambio desde el principio de su práctica profesional o necesitan empezar de forma tradicional y luego realizar un recorrido de toma de conciencia de la naturaleza cambiante de los procesos educativos? ¿Qué tienen que aprender los profesores noveles que van a integrar las TIC en su docencia? ¿Serán naturalmente, por su juventud y habilidades, innovadores integrando con interés las TIC en su enseñanza? ¿Qué condiciones se tienen que producir para que los procesos de cambio con Tic sean una prioridad en su trabajo?

\section{LAS TIC EN LA ENSEÑANZA UNIVERSITARIA Y LOS PROFESORES NOVELES}

Como se ha indicado, el estudio sobre la presencia, pertinencia, interés, características e innovaciones realizadas con las TIC en la enseñanza universitaria es ya un clásico reciente. La universidad es sujeto de modernización y transformación. Las TIC forman parte de una innovación que renueva las metodologías, o que desafortunadamente se puede quedar en una estrategia de reinstrumentalización de sus docentes.

Mirando en otros contextos para aprender de su evolución observamos que los espacios no universitarios aparecen forjados por la actividad de los docentes pero también por la regulación administrativa y curricular y las políticas educativas -recientemente las políticas enemigas de lo público-, por las multinacionales, como dirían los promotores del software libre, y por la opinión pública, como diría Senge (2002); por lo que las dificultades de la integración de las TIC en educación están bien presentes: 30 años de intento de introducción han tenido muy pobres resultados. Algunos procesos son, además, de reinstrumentalización de la función docente, como ocurre con cierto empeño periódico por adoptar algún tipo de hardware o software.

En la universidad se disfruta de cierta libertad de las constricciones burocráticas y curriculares y, de alguna forma, de las políticas. Es un espacio en teoría excelente para la innovación y la transformación de muchos aspectos de la enseñanza practicada. Creemos que queda espacio para procurar innovación en la universidad (Michavila, 2002; Suárez, 2004; Valcárcel, 2003).

La práctica ofrece, es verdad, una progresiva burocratización de las tareas de los docentes universitarios, y las políticas de rendición de cuentas condicionan notablemente la orientación profesional de los docentes. La ausencia de sustrato educativo, el peso del grupo especializado de investigación sobre el grupo interdepartamental de docencia y la génesis individualista de la profesión de docente universitario imponen una visión muy tradicional y fragmentada de la enseñanza, que por sí sola bloquea cualquier proceso de cambio, pongamos por caso la integración de las Tic. 
Si, tal y como plantea Somekh (2008), la innovación con TIC requiere estrategias holísticas en la enseñanza, y no es una técnica, los docentes universitarios no se encuentran en buenas condiciones para adoptarlas.

La génesis de la profesión docente universitaria, sin ser el único factor que construye esta visión tradicional y fragmentaria de la docencia, es un factor explicativo, pues el colectivo de docentes noveles accede primero a la función docente y luego se puede llegar a formar, siempre que su universidad disponga de algún plan para ello. No hay formación, hay una colección de técnicas.

En algunas universidades se están poniendo en marcha programas para profesores noveles. Estos programas tienen un currículo elemental, una formación básica. Por ejemplo, en la UAM (http://www.lauam.es/vicerrectorado/formacion_docente/ publico/index.php) el programa se desarrolla con cuatro grandes bloques, denominados: Habilidades docentes básicas, Metodologías docentes, Evaluación del aprendizaje y Tecnologías de la información, con módulos en cada uno de ellos, por un total de unas 150 horas de formación presencial y 300 de trabajo individual. El investigador participa en un módulo dentro del último bloque temático.

La oportunidad, sentido y valor de iniciativas de formación inicial como ésta es enorme, si bien no se nos escapa que adolecen de cierta simplicidad y alguna insuficiencia en el planteamiento. Responde a la misma lógica de técnicas de la que ha venido adoleciendo la formación continua en las universidades.

Los participantes, nos lo dicen ellos mismos, realizan la formación dentro de la lógica del mérito necesario para incorporarse al sistema. Aunque "lo importante es aprender", se busca con deseo el certificado de cada módulo.

¿Qué otra docencia se debería plantear? La percibimos como una enseñanza más abierta, aunque sus características están difusas en la literatura. En el campo de la tecnología educativa ha sido necesario redefinir algunos aspectos de la enseñanza que es así entendida, y nos puede servir como analogía para entender este nuevo espacio. Así, McCombs y Vakili (2005) mencionan implicaciones específicas para crear enseñanza en entornos en línea centrados en el alumno, listados de acciones y herramientas o actividades con herramientas:

- Desarrollar alfabetización digital vinculada a estrategias de pensamiento de alto nivel mediante búsqueda, cuestionamiento y descubrimiento de una variedad de recursos, fuentes y usos apropiados de la información obtenida vía electrónica.

- Proporcionar acceso a datos reales, a expertos en los campos de estudio y a tareas relevantes del mundo real (bien con documentos auténticos, bien con medios de comunicación síncrona y asíncrona).

- Ofrecer medios (como blogs, portafolios, autoevaluaciones) para aprender mediante reflexión.

- Involucrar activamente a los estudiantes en la discusión de casos, la solución de problemas, la participación en proyectos y actividades, que permitan la colaboración, el diálogo y la construcción del propio 
conocimiento (proyectos colaborativos en comunidades web, viajes virtuales, webquests).

- Propiciar oportunidades de contacto personal y desarrollar actividades en línea con fines tanto académicos como comunicativos y sociales para fomentar un sentido de comunidad y reducir los sentimientos de aislamiento (foros y chats académicos; videoconferencias interactivas; círculos de estudio; clubes y redes estudiantiles; torneos y juegos en línea; libros y álbumes electrónicos elaborados por los grupos de alumnos; servicio electrónico de mensajes sociales; blogs y wikis propuestos por los estudiantes).

- Permitir que los estudiantes con apoyo de sus asesores elaboren portafolios electrónicos y otro tipo de evaluaciones auténticas que den cuenta del nivel de logro y habilidades adquiridas, e introducir recursos de autoevaluación y rúbricas que permitan definir y evaluar no sólo los aspectos cuantitativos, sino cualitativos de la participación y el aprendizaje en línea.

- Proporcionar una realimentación y evaluación continua al estudiante, así como la oportunidad de tomar decisiones y elegir alternativas en un ambiente estimulante que le proponga desafíos constantes, pero abordables.

- Atender a las diferencias y necesidades individuales de los estudiantes, con apoyo en materiales instruccionales en formatos electrónicos no lineales, con la opción de optar por múltiples caminos a través de textos, gráficos, vídeo, animación, etcétera, y prever trayectorias flexibles y con las adaptaciones curriculares apropiadas para estudiantes con capacidades diferentes.

- Considerar distintos niveles de tutoría y asesoría, así como formas de organización y participación en la comunidad de aprendizaje en línea, en función de las necesidades, intereses y avance mostrados por los participantes.

Se trata de una concepción ampliada de la enseñanza y la búsqueda de herramientas que lo hacen posible.

Para poner en marcha este tipo de enseñanza, algunos autores como Gairín (2009) proponen relacionar dominios de innovación pedagógica (una didáctica de la innovación) y niveles de innovación (asimilativo, en transición, para la transformación). El horizonte de los docentes noveles sería una enseñanza que se preocupa por una participación activa en la construcción de conocimiento que, a nuestro juicio, puede estar o no soportada por determinadas herramientas o soluciones técnicas. Lo importante es el planteamiento de una enseñanza ampliada que da sentido a determinadas actividades que integran TIC.

En los análisis sobre la adscripción de determinadas herramientas a prácticas de enseñanza, su mayor mérito quizá sea identificar la naturaleza de las propias prácticas de enseñanza (expositiva, de inmersión tecnológica, de indagación, de 
colaboración y realización de proyectos, de resolución de problemas, de generación de conocimiento), más que las posibilidades "intrínsecas" de las herramientas (búsqueda, presentación, comunicación, colaboración, construcción de conocimiento, organización semántica, modelado dinámico, interpretación visual).

Este tipo de enfoque sobre posibilidades propone una lectura de que quien incluye determinada TIC apuesta por determinada práctica de enseñanza. Recoge la idea de affordance o "posibilidad latente" de las Tic, tan querida a los tecnofílicos en general. Con tales presupuestos, una formación basada en técnicas tendría un enorme éxito en la transformación de las prácticas de enseñanza.

Nosotros defendemos, como luego se dirá, que el camino posiblemente sólo tenga una dirección, que sólo desde determinadas concepciones abiertas de enseñanza es posible una integración de las Tic que resulte de provecho al aprendizaje autónomo de los estudiantes. Con ayuda de los estudiantes noveles se puede valorar ese recorrido, de las teorías a las prácticas y de los usos a las teorías.

Sólo recientemente está interesando el estudio de las prácticas docentes de los profesores noveles universitarios, ligado a los estudios de género, y el impacto que supone vivir en un espacio cambiante por las reformas universitarias y las condiciones de trabajo (colectivo CECADE, http://cecace.org/proj-profuni.html). La metodología que se utiliza es la autobiografía triangulada mediante resonancias o conexiones metafóricas. Se trata de una promisoria línea, que supone una presencia larga del investigador en el campo y una implicación del propio docente novel en un proceso indagatorio, con conciencia sobre los procesos de cambio que se producen al realizar estos procesos de indagación.

Otro abordaje son análisis de la enseñanza con y sin TIC, una investigación preocupada por los usos y las teorías implícitas y creencias de los sujetos. Se realiza con protocolos y rejillas. La ventaja de un protocolo es su flexibilidad de aplicación, su proximidad al diseño de la propia enseñanza, y que con el mismo se dibuja un universo de creencias sobre la enseñanza practicada y los conflictos, las dudas y la decisión de cambiar el tipo de enseñanza practicada, algo conocido tradicionalmente en las técnicas de encuesta. González (2009) identifica y adapta diversos instrumentos para analizar la integración de las TIC en la enseñanza on line desde la perspectiva del profesor (lo que piensa, sus conductas). Entre las variables que valora en una enseñanza centrada en el estudiante están el papel de las TIC para:

- Motivar a los estudiantes de forma intrínseca.

- Descubrir y construir conocimiento.

- Utilizar la experiencia de los estudiantes.

- Atender a los estudiantes.

- Evaluar de forma más flexible y diversa, autoevaluar.

- Identificar y remediar dificultades de estudio de los estudiantes.

- Proveer recursos.

- Utilizar la web de forma síncrona.

- Utilizar la web para el establecimiento de vínculos dentro del grupo.

- Generar trabajo grupal con análisis, diálogo y reflexión. 
Las respuestas ofrecidas por los docentes ayudan a dibujar un espacio de usos de las TIC y, por ende, una manera de concebir la enseñanza.

En la aplicación de protocolos sobre la enseñanza con TIC suelen producirse dificultades, por falta de reflexión sobre la propia naturaleza de la enseñanza y cierta idealización de los atributos de las Tic introducidas.

Aparece, pues, la idea de affordance o "posibilidad latente" de las TIC, antes señalada, que ha sido valorada por Somekh (2008). Los profesores necesitan continuo apoyo para hacer usos creativos de las posibilidades latentes de sus herramientas. Si no, sus prácticas serán conducidas por las rutinas. Somekh recomienda reorientar los patrones de práctica de los docentes y sus relaciones con los estudiantes (preguntarse por cuál es el estatus de los estudiantes, lo que equivale a pensar en las prácticas de enseñanza que se emprenden).

Los protocolos no dejan indiferentes a los docentes que entrevistamos, y nos interesan principalmente aquellos que se cuestionan la forma en que trabajan. Así, en aquellos elementos en los que el docente duda, se cuestiona o está abierto es posible avanzar hacia prácticas más ricas con Tic, lo que nos parecía de enorme interés para profesores noveles y su capacidad de ideación de nuevos entornos de aprendizaje.

Esta cuestión de la falta de certidumbres y el deseo de cambio (en el que las TIC eran un motivo para ello) es el espacio de indagación que más nos atraía analizar entre los noveles.

Dice Dede (en Hanna, 2002) que el profesor está intentando dominar la educación virtual considerándola erróneamente como una variante secundaria de la educación convencional en lugar de los procesos y redes que transformarán nuestra civilización. Esto puede ser particularmente cierto en la enseñanza universitaria. Pero los errores de los docentes no se apoyan en su percepción de las posibilidades de las TIC, sino de la concepción de la propia enseñanza. Tal percepción de las TIC, en cualquier caso, será lo que venga a reafirmar un tipo de prácticas de enseñanza.

\section{Metodología del ESTUdio}

Los objetivos del estudio fueron caracterizar la percepción del trabajo como docentes de los profesores noveles universitarios, para valorar la naturaleza de su práctica profesional; así como identificar de qué forma van a integrar las Tic en su docencia.

Se trata de un estudio exploratorio descriptivo en el que se examina su percepción de su práctica con TIC como docentes mediante un protocolo específico y un proceso formativo monitoreado en una plataforma de teleformación, que podríamos caracterizar en su conjunto como observación participante con recogida de evidencias en forma de productos. 
La muestra consistió en 26 profesores noveles de una misma universidad que participan voluntariamente en un proceso de formación inicial modular de una universidad pública madrileña, donde cada módulo obtenido se recompensa con una acreditación como formación recibida. Sólo 9 de ellos son hombres. Los profesores noveles pertenecen a 4 colectivos: Ciencias Experimentales, Ingeniería, Ciencias Sociales y Humanidades. No hay representantes del campo de las Ciencias Médicas.

Los principales resultados están relacionados con la aplicación del protocolo previo, el desarrollo de un curso o módulo y con el miniproyecto desarrollado por los participantes en el mismo.

Los instrumentos utilizados fueron un protocolo específico generado en un estudio de casos previo (Barros, Chavarría y Paredes, 2008), interacciones dentro de un curso apoyado por una plataforma, la valoración por el profesor de una tarea compleja encargada a los participantes (un miniproyecto), comentarios informales (durante un curso, en la tutela del proyecto) y valoración de su utilización de las herramientas TIC en una plataforma Moodle.

En el protocolo (con una escala 0-4, no-poco-bastante-mucho) se plantea el análisis de:

- Relaciones entre concepciones de enseñanza y prácticas de enseñanza.

- $\quad$ TIC utilizadas y usos de las TIC en la docencia universitaria.

- Reforzamiento de prácticas tradicionales con TIC.

- Impacto de las Tic en los aprendizajes de los estudiantes.

- Prácticas de enseñanza sin certidumbres o abiertas.

- Docentes que están en el camino del cambio de los usos transmisores de las TIC hacia prácticas más abiertas.

El protocolo fue aplicado para provocar en los participantes aspectos relevantes sobre sus prácticas de enseñanza, con idea de que pudieran valorar mejor el papel de la plataforma y de algunas funcionalidades de la misma en sus propias prácticas.

Para entender el análisis posterior de resultados, conviene indicar que el módulo formativo previo a la recogida de datos atendía el uso de una plataforma de teleformación, Moodle, en procesos de blended learning, desde una perspectiva centrada en la enseñanza (analizando con detalle aspectos de planificación que deberían ser tenidos en cuenta para implementar el uso de la plataforma y presentando a continuación las principales posibilidades de la herramienta, pero sin dar importancia a una descripción exhaustiva de las funcionalidades de la misma). Tenía una parte presencial, de 11 horas, y una parte a distancia con la realización de diversas actividades y un miniproyecto, de 3 meses, con una tutela ECTs, y una valoración del trabajo.

Se generaron algunas situaciones que permitieron recoger un conjunto de evidencias sobre las que elaborar una explicación de conjunto sobre el tipo de disposición de los futuros docentes a la integración de las Tic. La acción propuesta a los 
participantes del curso, el miniproyecto, es un ejercicio deliberado de transformación de sus percepciones sobre las TIC en educación, pero no puede suponer un cambio radical, por su naturaleza concreta y limitada en el tiempo; se convierte más bien en un espacio para dialogar, investigar y crecer.

En relación con la temporalización del estudio, se indica que hubo una recogida constante de información a lo largo de un curso académico. De forma principal, se interrogó a docentes noveles mediante el protocolo citado durante el desarrollo del proceso de formación inicial modular, y se verificó el alcance de la integración de las TIC cuando los participantes concretaron en un miniproyecto qué tipo de integración hacían.

\section{DisCUSIÓN DE RESULTAdOS}

Al final del proceso, tras desarrollar el miniproyecto cada participante, se observa en la evaluación del mismo que los docentes noveles que participan en este estudio apuntan las maneras de muchos docentes en ejercicio (Hanna, 2002), que nos manifiestan de diversos modos: sentido de independencia y libertad, capacidad de organizar de manera autónoma sus obligaciones (entre las que está la docencia), fuerte orientación a la especialización, escasa percepción de conjunto (sea su departamento, Titulación, Facultad, Universidad), escaso interés por revisar la perspectiva académica de otros colegas (de su Departamento, de Departamentos afines en su Universidad o en otras). De manera complementaria a este proyecto conviene indicar que en diversos comentarios informales nos manifiestan tensión por la carrera universitaria (sus acreditaciones, sus publicaciones), sobre todo cuando justifican su escasa implicación en el proyecto desarrollado. No muestran tampoco una particular implicación en la enseñanza que practican (sólo una participante de 26 decidió utilizar lo aprendido para implementar un curso), ni utilizan guías didácticas ajenas para contextualizar sus ejercicios, ni conocen las guías docentes que han aprobado en sus respectivos departamentos, ni tienen dudas pues sólo cinco de ellos acuden a una sesión con formato de tutoría ECTS de preparación de los materiales que vuelcan en la plataforma.

No hay pues condiciones iniciales para reflexionar sobre la enseñanza, ni para fundamentar la integración de las Tic en un proceso de trabajo en equipo o una indagación colaborativa.

Veamos entonces qué elementos pueden estar contribuyendo a la dirección que toma su percepción sostenida sobre las TIC en educación universitaria.

A los docentes noveles que han comenzado a enseñar en la Universidad no les preocupa demasiado la transformación metodológica que supone la utilización de las TIC como una herramienta para innovar en la docencia universitaria, toda vez que, como se muestra la Tabla 1, no la visualizan inicialmente en una docencia universitaria regular, donde ellos se desempeñan, sino más bien en otros espacios y con otros tiempos (al preguntarles durante el curso sobre el sentido o utilidad de 
la formación que estaban recibiendo). En la Tabla 1 se muestra la disposición inicial de los docentes noveles hacia la inclusión de unas TIC concretas, como las plataformas de teleformación, en los procesos de enseñanza que van a emprender. Muestra una saturación de respuestas sobre cómo integrarán las Tic, en este caso el uso de una plataforma, en espacios alejados de lo regular. Sólo uno de cada tres percibe al principio de su formación que pueda utilizarse una plataforma de teleformación, que es una propuesta de enseñanza, en un curso regular de Grado (Tabla 1).

\section{TABla 1. PERCEPCiÓN PREVIA DEL USO DE LAS PlatAFORMAS EN EDUCACiÓN UNIVERSITARIA (TIPO DE CURSOS EN LOS QUE SE PUEDE APLICAR) POR PARTE DE DOCENTES NOVELES}

\begin{tabular}{|c|c|c|c|c|c|}
\hline & $\begin{array}{c}\text { No las } \\
\text { aplicará }\end{array}$ & En Grado & En Posgrado & $\begin{array}{c}\text { En materias } \\
\text { optativas }\end{array}$ & $\begin{array}{c}\text { En otras } \\
\text { actividades } \\
\text { formativas }\end{array}$ \\
\hline $\mathrm{N}$ & 1 & 8 & 6 & 1 & 9 \\
\hline$\%$ & 4 & 32 & 24 & 4 & 36 \\
\hline
\end{tabular}

Esta constatación rompe de alguna forma con el mito de que nuevas generaciones de docentes abrazan nuevos métodos por novedad, contagio o simpatía. Aquí no ocurre así.

La enseñanza para los profesores noveles es más bien un espacio con unas reglas, que ya han aprendido por implosión y resolviendo con su propio juicio, no reflexionando por sí y con otros.

Las TIC no son una prioridad en la enseñanza que practican los profesores noveles. Puesto que la introducción de las Tic obedece a una lógica innovadora (Sancho, 2006), la innovación no es una prioridad para el colectivo en formación.

Tal y como nos confiesan dos de los participantes en el curso en una conversación informal, están más cerca de la lógica de la producción científica de su área de conocimiento, la manera de ser docente universitario que más réditos profesionales y prestigio procura.

¿Hay certidumbres? ¿Hay dudas? ¿Hay incoherencias? ¿Qué podemos colegir en estas declaraciones? ¿Qué diferencias hay entre áreas de conocimiento? La necesidad de utilizar herramientas TIC en todos los campos de conocimiento va marcando una exigencia en la enseñanza. ¿Los estudiantes reconstruyen conocimiento con las TIC?

Las respuestas que ofrecen los estudiantes al protocolo se sintetizan en la Tabla 2 y la Figura 1. Algunas respuestas abiertas se presentan a continuación, señalando al profesor participante entre paréntesis.

En primer lugar, en los resultados no observamos que desde ningún área de conocimiento (ni siquiera con una burda división Ciencias-Letras) aparezca una inclinación hacia alguna temática metodológica o de integración de las Tic que se plantea en el protocolo. 
Va calando el uso de Internet por parte de los investigadores como herramienta de trabajo, y eso se empieza a llevar a las clases. Alguno incluso lo considera desde la perspectiva de las competencias profesionales que deben adquirir los estudiantes: "Trato de que usen nuevas tecnologías en la asignatura orientado a desarrollar sus competencias laborales" (Prof. 7).

No hay un comportamiento de "nativos digitales" entre los profesores noveles. Treinta años después de la aparición de los microcomputadores los noveles hacen a priori una interpretación corta de las posibilidades de la ampliación del conocimiento en la enseñanza mediante herramientas diversas. Sólo después de enfrentarse con las herramientas y reflexionar sobre su propia enseñanza comenzarán a dudar, como luego se dirá.

La mayoría de los nuevos docentes utiliza bases de datos e Internet. Algunos, muy pocos, conocen páginas web o material digital de utilidad para su profesión y su actividad investigadora. Otros pocos conocen las simulaciones y representaciones animadas.

Lo digital no es sinónimo para ellos, por tanto, de conocimiento devaluado. Pero el grueso de la información científica está aún en los libros.

Casi todos hacen usos genéricos de las TiC relacionados con búsquedas. Casi todos valoran que los estudiantes busquen. Algunos valoran la búsqueda en bases de datos específicas. Unos pocos de ésos las incluyen como referencia de estudio para sus alumnos.

Como se aconseja su uso en clase, se está realizando una alfabetización en competencias genéricas de búsqueda de información.

A pesar de todo, el uso de Internet por parte de los profesores, que se convierte en una condición del trabajo de un investigador, no es necesariamente implicar a los estudiantes en vivenciar los procesos de investigación de los propios docentes. Es habitual que utilicen la web como repositorio: "La web docente me ha servido de gran ayuda" (Prof. 13).

La enseñanza universitaria está cambiando. Es más compleja que en fechas recientes. Afortunadamente, en los últimos años las prácticas de diversa índole forman parte de la formación que reciben todos los universitarios, lo que modifica el panorama de transmisión de saberes que ha sido la universidad.

Los profesores noveles, a tenor de sus respuestas, parecen haber vivido una enseñanza y no ser capaces de imaginar otra. Tienen una estructura de conocimiento pedagógico superficial, alguna idea central y otras pocas conectadas a esta idea (Marcelo, 2008). En general, no hay reflexión ni dudas. Parece que «todo depende del profesor" y "el profesor se hace a sí mismo", mitos que fundamentan la enseñanza más inmóvil y tradicional. Sólo una profesora novel, de Ingeniería química, reconoce: "En el aula no se puede aprender todo. En el trabajo (que tendrán estos estudiantes) tienes que seguir formándote autónomamente" (Prof. 24).

Todos los aspectos de metodología sugeridos de forma sistemática en el protocolo de entrevista les parecen adecuados a los profesores noveles, aunque los tienen en poca estima (Tabla 2 y Figura 1). 
JOAQUÍN PAREDES LABRA

DOCENTES NOVELES UNIVERSITARIOS Y SU ENSEÑANZA CON TIC

TABla 2. Aspectos De la ENSEÑANZA PRACTICADA POR DOCENTES NOVElES (RESPUESTAS EN El PROTOCOlO)

\begin{tabular}{|c|c|c|c|c|c|}
\hline Bloques & Preguntas & no & poco & bast. & mucho \\
\hline \multirow{3}{*}{ TIC en asignaturas } & recursos para estudiantes & 2 & 5 & 9 & 2 \\
\cline { 2 - 6 } & búsqueda & 2 & 8 & 6 & 2 \\
\cline { 2 - 6 } & entorno & 2 & 8 & 6 & 2 \\
\hline \multirow{5}{*}{ Docencia con TIC } & material de interés para profesor & 4 & 7 & 5 & 2 \\
\cline { 2 - 6 } & $\begin{array}{c}\text { actividades con } \\
\text { materiales diversos }\end{array}$ & 2 & 10 & 3 & 3 \\
\cline { 2 - 6 } & extensión programa & 8 & 5 & 2 & 3 \\
\cline { 2 - 6 } & carga trabajo & 3 & 9 & 4 & 2 \\
\cline { 2 - 6 } & documentos especiales & 6 & 8 & 3 & 1 \\
\cline { 2 - 6 } & resolución colectiva & 4 & 7 & 3 & 4 \\
\cline { 2 - 6 } & tutorías & 4 & 6 & 7 & 1 \\
\cline { 2 - 6 } & evaluación & 4 & 6 & 7 & 1 \\
\hline
\end{tabular}

Figura 1. ASPECTOS DE LA ENSEÑANZA PRACTICADA POR DOCENTES NOVELES (RESPUESTAS EN El PROTOCOlO)

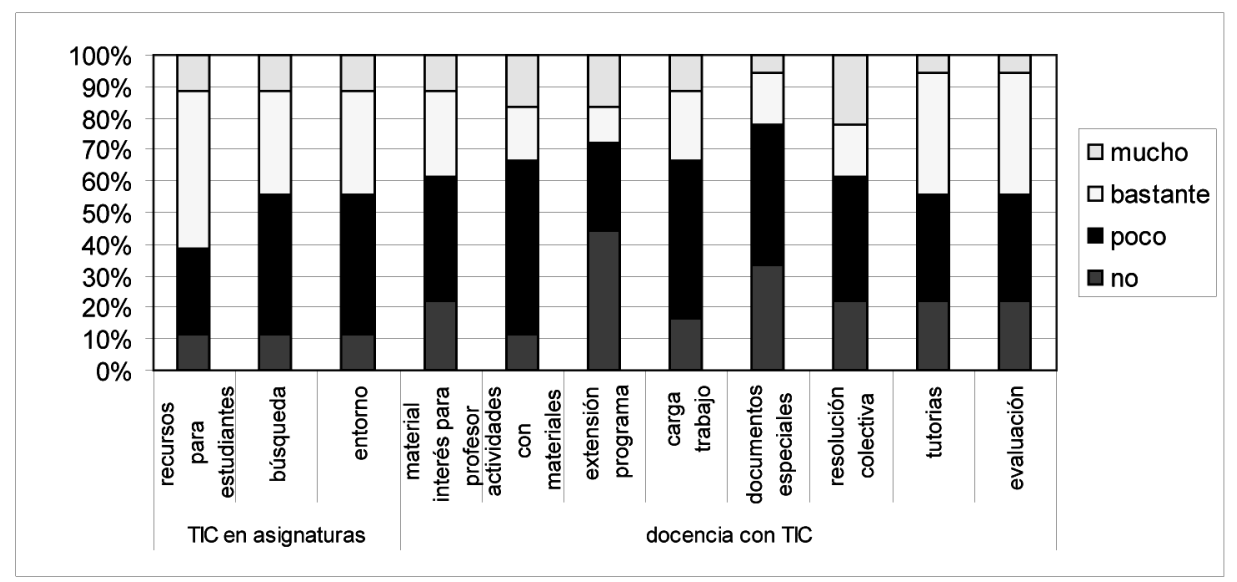

Al responder al protocolo se acuerdan de todos los aspectos de la enseñanza, que a su vez han ido conociendo en sucesivos módulos de formación inicial. Sin embargo, no los asumen ni los aceptan, al menos con los procesos que han vivido hasta ahora, van contra su percepción de la enseñanza o la forma en que van resolviéndola. Posiblemente faltan más procesos reflexivos. En general es raro encontrar reflexión sobre la propia práctica. 
Como dice uno de los profesores: "Probablemente no sea estrictamente necesario, pero sí altamente eficaz" (Prof. 21). La eficacia es una constante clásica entre los noveles, resolver la docencia con eficacia parece ser la principal meta.

Algunos hacen aflorar la coherencia de lo que hacen con un esquema tradicional de enseñanza con presentación, actividad de refrendo, sanción de la misma, examen. Al referirse al trabajo en grupo, a uno de los noveles le preocupa la valoración individual del mismo: "Es muy difícil precisar si los alumnos de un grupo han trabajado todos con la misma intensidad" (Profesor 13).

Un importante grupo de profesores sienten inclinación por presentar toda o la mayor parte de la información relevante. Están poco dispuestos a flexibilizar los contenidos de sus programas para facilitar tiempo a los estudiantes para investigar por su cuenta. Creen incluso que una enseñanza centrada en contenidos es un signo o "punto fundamental de la calidad de la docencia" (Prof. 15), que "el sistema perfecto sería que, sin renunciar a los contenidos (las TIC), nos permitieran amenizar el aprendizaje" (Prof. 16).

Otros piensan que las competencias genéricas como el estudio y el trabajo del estudiante las enseñan otros, pero no ellos.

Tienen en alta consideración el papel de las tutorías y formas evaluativas diversificadas (Figura 1). Pero la tutoría y la evaluación no contribuyen a la reconstrucción del conocimiento, sino al control. Sin la presencia de la reflexión entre los estudiantes, o de las dudas entre los noveles, no tiene mucho interés la apertura a nuevas formas de enseñanza.

Algunos profesores, como unas profesoras noveles de psicología, tienen una visión abierta de la metodología de enseñanza, pero no consideran aspectos de tutoría y evaluación. Son, por esa razón, tradicionales y eclécticas, también en cuanto a la integración de las TIC (usar la web como banco de información, uso selecto de determinadas páginas); además están poco comprometidas con la integración de las TiC en su enseñanza.

No aparece entre los noveles el compromiso real con una enseñanza más variada y con más dimensiones, que debería suponer una perspectiva para la construcción de conocimiento por parte de los estudiantes.

Quizá el eclecticismo en la concepción de la enseñanza es responsable de que los usos de las TIC y su integración en la enseñanza se entrevean tan pobres.

Este protocolo nos ayuda, entonces, a ver las escasas posibilidades para la integración de las TIC en la enseñanza que practiquen estos docentes noveles por la dificultad de organizar una enseñanza más abierta, con más actividades y recursos; en todo caso, a pesar de tantas declaraciones por parte de los noveles sobre la bondad de la importancia de la resolución de problemas, la realización de prácticas, el seguimiento individualizado de los estudiantes, la evaluación continua y la diversidad de actividades de evaluación, las actividades con Tic (dentro o fuera de una plataforma de teleformación) estarán presentes como meras actividades complementarias. 
Este eclecticismo no existe entre profesores con experiencia. Si son tradicionales, no diversifican ni la metodología ni la evaluación, ni perciben que las TIC sean herramientas para construir conocimiento. Si tienen dudas, comienza su diversificación de la enseñanza y el papel de las Tic va más allá de la presentación de información (por ejemplo Gewerc, 2008).

Al respecto, conviene indicar que tras el módulo, sólo una de las 26 profesoras noveles solicitó abrir un curso completo en la plataforma Moodle para desarrollar su actividad docente. Fue una profesora de Ciencias la que, a tenor de sus respuestas a la pregunta recogida en la Tabla 1 y al protocolo, sin perspectiva previa sobre las posibilidades de las TIC y sin conocer recursos TIC, tiene una perspectiva más dialógica de la enseñanza ("la interacción entre los estudiantes, para que se den cuenta de sus puntos débiles", Prof. 7) y propone variedad (como por ejemplo producir materiales mediante trabajo en grupo). El resto hicieron una práctica corta, con algunas actividades.

A ella se añaden otros dos profesores, una de Ciencias y otro de Políticas, que tienen una perspectiva más abierta de la enseñanza y que apuesta por los usos integrados de las TIC en sus prácticas ("Los estudiantes van a variar su forma de plantear el estudio en función del tipo de evaluación", Prof. 24).

Tras el curso y sus prácticas con una plataforma, Moodle, les preguntamos si se habían replanteado su trabajo como docentes. La mayoría, cuatro de cada cinco, manifiestan haberlo hecho, y confesaban que se habían replanteado la naturaleza del aprendizaje, la importancia concedida a los contenidos, la metodología de su enseñanza... A algunos se les abrieron interrogantes y dudas. Este resultado es muy esperanzador, que una enseñanza más problematizadora, que permitió ver qué hacían otros compañeros, que planteó dudas y preguntas durante el proceso, que ofreció formación y buscó soluciones, que acompañó una parte de su cambio, tuvo como resultado algunas dudas e interrogantes sobre aspectos que van más allá de la transmisión de la enseñanza.

Otras preguntas para estudios que den continuidad a éste serían las siguientes: teniendo en cuenta que la enseñanza es modular y para llegar a conocer las plataformas ya han pasado por los fundamentos y técnicas de enseñanza y entran en los recursos, ¿está mal fundamentada la naturaleza de la enseñanza en la formación inicial que reciben los docentes noveles? ¿Hay otros factores que inciden en los docentes noveles en la forma de concebir la enseñanza, como biografía, prioridades en la carrera investigadora, inexistencia de trabajo en equipo en el seno de sus titulaciones...? ¿Qué pasaría si hubiese equipos en vez de individuos, diseñando estos materiales y cursos? ¿Qué pasaría si hubiese un proceso de indagación sobre las propias prácticas? Sospechamos que habría más oportunidades de comenzar una enseñanza más abierta que integra Tic, como una manifestación de una enseñanza comprometida e innovadora. 


\section{CONCLUSIONES E IMPLICACIONES PRÁCTICAS}

A los profesores noveles no les interesa la universidad excelente que defiende Hanna (2002), preocupada por la rendición de cuentas y la dirección eficaz, algo para los políticos de la educación y los docentes volcados en la política institucional, que a su vez es el acicate para la transformación metodológica de las universidades y sus decisiones institucionales asociadas; sino el propio sistema de rendición de cuentas académico en forma de artículos científicos reunidos en revistas de impacto.

Esta forma de política universitaria es una constricción para la práctica de una enseñanza diferente. Quizá también debería ser un aviso para las percepciones voluntaristas de la transformación y el cambio de la docencia en la universidad.

A los noveles les interesa una enseñanza que funcione, que no es precisamente una enseñanza que integra TIC sino la que se reinstrumentaliza con TIC. Adoptan, eso sí, los usos de las TiC que han ido calando como contenidos de la formación de profesionales.

Por el contrario, una práctica docente contextualizada, en equipo, indagatoria, con formación, parece contribuir a una enseñanza más plural y que integra TIC.

Ciertamente, los sistemas de acreditación comienzan a valorar la participación en procesos de formación e innovación, pero ¿sería provechoso para la universidad del futuro que la carrera docente tuviera en consideración las prácticas docentes con indicadores más diversos? Esperemos que esta propuesta de pluralidad no contribuya a que la perversión de los sistemas de acreditación acabe haciendo más complicada la ya de por sí tortuosa incorporación de nuevos docentes a las universidades.

Como perspectiva de futuro, para los docentes noveles en la universidad la enseñanza no puede ser una colección de habilidades docentes ni los procedimientos de acceso la mera implosión, sino que deben ampliarse las bases de preparación, debe incorporarse una práctica guiada y algún proceso reflexivo de los que recomienda el movimiento de indagación (coaching, pares, indagación reflexiva, investigación acción, comunidades de práctica...).

En cualquier caso, se debe cuidar precisamente esta fase, de inserción, mediante orientación, mentoría, formación, colaboración con otros docentes, reducción laboral, diálogo con colegas... (Marcelo, 2008), algo que ahora es un sueño.

Las políticas llegan a la universidad (otra vez los neoliberales), pero no llegan a las prácticas de enseñanza universitaria, o llegan pero en direcciones poco interesadas en las propias prácticas. Para paliarlo convienen políticas de mesonivel, que indica Tondeur (2008), sobre políticas sobre TIC en los centros universitarios, como proyectos TIC (apoyo, evaluación, cooperación relacionada con las TIC), infraestructuras TIC en el centro, y actuaciones en el micronivel.

Sobre estas condiciones quizá sea posible plantear el desarrollo de una innovación como la adopción de TIC, pues requiere la promoción de un proceso de cambio, que tiene que ver, según Somekh (2008), con un deseo por impulsar 
aprendizaje situado, valorar la propia práctica, una comunidad de práctica y un nuevo valor al conocimiento generado por los estudiantes.

Las TiC se convierten en un epifenómeno, agobiados por la urgencia de resolver la enseñanza, o sin haber resuelto primero qué tipo de enseñanza deseamos.

\section{REFERENCIAS BIBLIOGRÁFICAS}

AlBA, C. (2005) Viabilidad de las propuestas metodológicas derivadas de la aplicación del crédito europeo por parte del profesorado de las universidades españolas, vinculadas a la utilización de las TIC en la docencia y la investigación. Madrid, Dirección General de Universidades. Consultado el 1 de junio de 2010. http://www.mec.es/univ/html/informes/ estudios_analisis/resultados_2004/ea0042/EA-2004-0042-ALBA-2-InformeGlobal.pdf.

Barros, B.; Chavarría, M. y Paredes, J. (2008) Para analizar la transformación con TiC de la enseñanza universitaria. Un estudio exploratorio sobre creencias pedagógicas y prácticas de enseñanza con TiC en universidades latinoamericanas. Revista Interuniversitaria de Formación del Profesorado, 9 (1). Consultado el 1 de junio de 2010. http://www. aufop.com/aufop/home.

BROwn, M. (2008) Interactive learning environments: review of an old construct with a new critical twist, en Voogt, J. y KNEZEK, G. (eds.) International handbook of information technology in Primary and Secondary Education. Nueva York, Springer, 231-248.

FERnÁNDEZ PÉREZ, M. (1989) Así enseña nuestra Universidad hacia la construcción crítica de una didáctica universitaria. Madrid, Universidad Complutense.

Gairín, J. (2009) Cambio y mejora: la innovación en el aula, equipo de profesores y centro. En PARedes, J. y HerRÁN, A. (coords.) La práctica de la innovación educativa. Madrid, Pirámide, pp. 21-48.

GarCía-VAlCÁrcel, A. (coord.) (2001) Didáctica universitaria. Madrid, La Muralla.

Gadotti, M. (2005) Historia de las ideas pedagógicas. México-Buenos Aires, Siglo XxI.

GEWERC, A. (coord.) (2008) Modelos de enseñanza y aprendizaje presentes en los usos de plataformas de e-learning en universidades españolas y propuestas de desarrollo. Informe final. Madrid, Dirección General de Universidades.

GONZÁLEZ, C. (2009) Conceptions of, and approaches to, teaching online: a study of lecturers teaching postgraduate distance courses. Higher Education, 57, 299-314.

Hanna, D. (ed.) (2002) La enseñanza universitaria en la era digital. Barcelona, Octaedro.

MARCELO, C. (coord.) (2008) El profesorado principiante. Inserción a la docencia. Barcelona, Octaedro.

McCombs, B. y VAKILI, D. (2005) Learner-Centered Framework for E-Learning. Teachers College Record, 107 (8), 1582-1600.

Michavila, F. y MARTíNez, J. (2002) El carácter transversal en la educación universitaria. Madrid, Dirección General de Universidades

SANCHO J. (coord.) (2006) Tecnologías para transformar la educación. Barcelona, UNIAAkal.

SENGE, P. (2002) Escuelas que aprenden. Buenos Aires: Norma.

SOMEKH, B. (2008) Factors affecting teachers' pedagogical adoption of ICT, en VoogT, J. y KNEZEK, G. (eds.) International handbook of information technology in Primary and Secondary Education. Nueva York, Springer, 449-460. 
SuÁrez Arroyo, B. (2004) El Espacio Europeo de Educación Superior: una visión desde un observatorio de privilegio. Fuentes: Revista de la Facultad de Ciencias de la Educación, 6, 23-34.

TONDEuR, J. y otros (2008) ICT Integration in the Classroom: Challenging the Potential of a School Policy. Computers \& Education, 51 (1), 212-223.

VALCÁRCEL, M. (2003) La preparación del profesorado universitario español para la convergencia europea en educación superior. Madrid, Dirección General de Universidades. Consultado el 1 de junio de 2010. http://wwwn.mec.es/univ/html/informes/estudios_ analisis/resultados_2003/EA2003_0040/informe_final.pdf.

VV. AA. (2000) Formación pedagógica del profesorado universitario en España. Revista Interuniversitaria de Formación del Profesorado, 38 monográfico, 15-102.

- (2009) Docencia universitaria virtual. RELATEC: Revista Latinoamericana de Tecnología Educativa, 7 (2). Consultado el 1 junio de 2010. http://campusvirtual.unex.es/cala/ editio/index.php?journal $=$ relatec\&page $=$ issue $\& o p=$ current.

Zabalza, M. A. (2003) Competencias docentes del profesor universitario. Madrid, Narcea. 